\title{
Therapeutic Potential of the Ranibizumab Port Delivery System in the Treatment of AMD: Evidence to Date
}

This article was published in the following Dove Press journal: Clinical Ophthalmology

\author{
Eric R Chen' \\ Peter K Kaiser ${ }^{2}$ \\ 'School of Medicine, Case Western \\ Reserve University, Cleveland, OH, USA; \\ ${ }^{2}$ Cole Eye Institute, Cleveland Clinic \\ Foundation, Cleveland, $\mathrm{OH}$, USA
}

\begin{abstract}
Age-related macular degeneration (AMD) is one of the leading causes of blindness in older adults worldwide. The advent of intravitreal neutralization of vascular endothelial growth factor (VEGF) has revolutionized the management of patients with neovascular AMD, but current anti-VEGF therapies carry a high threshold of patient burden. The ranibizumab port delivery system (PDS) is an implanted, refillable reservoir that continuously supplies the anti-VEGF medication ranibizumab directly into the vitreous, eliminating the need for frequent intravitreal injections. It has most recently been evaluated in the Phase II LADDER trial demonstrating the efficacy and safety of the PDS, with Phase III trials currently underway. This review presents both the promise and drawbacks of the PDS in the treatment of AMD from the current literature.
\end{abstract}

Keywords: neovascular age-related macular degeneration, ranibizumab, port delivery system, vascular endothelial growth factor

\section{Introduction}

Age-related macular degeneration (AMD) remains one of the most common causes of vision impairment and blindness in older adults worldwide. ${ }^{1-4}$ Neovascular AMD (nAMD) is an advanced stage characterized by choroidal neovascularization, leading to edema, bleeding, fibrosis, and subsequent functional deterioration. These clinical manifestations of nAMD are due to angiogenic and pro-inflammatory cytokines, most notably vascular endothelial growth factor (VEGF). ${ }^{5}$

One of the biggest shifts in the management and treatment of nAMD was the discovery of directly targeting VEGF suppression, which have rapidly become the firstline approach in managing nAMD over established treatments like photodynamic therapy. ${ }^{6}$ Currently, there are five available anti-VEGF treatments in the United States: pegaptanib (Macugen; Eyetech Pharmaceuticals, New York, New York), bevacizumab (Avastin; Genentech, San Francisco, CA, USA), ranibizumab (Lucentis; Genentech), aflibercept (Eylea; Regeneron, Tarrytown, New York), and brolucizumab (Beovu; Novartis, Basel, Switzerland). All of these medications are currently approved by the FDA for use in nAMD with exception of bevacizumab, which is utilized off-label.

Several studies demonstrated the promise of neutralizing VEGF in nAMD, including a clinical trial demonstrating intravitreal pegaptanib delaying loss of vision and small studies describing visual improvement in patients treated with bevacizumab. ${ }^{7-9}$ However, it was not until the MARINA (Minimally Classic/Occult Trial of the
Correspondence: Peter K Kaiser Cole Eye Institute, Cleveland Clinic Foundation, 9500 Euclid Avenue, Desk i3125, Cleveland, $\mathrm{OH} 44195$, USA

$\mathrm{Tel}+\mathrm{I}(2 \mathrm{I}$ ) 444-6702

Email kaiserp@ccf.org 
Anti-VEGF Antibody Ranibizumab in the Treatment of Neovascular AMD) and ANCHOR (Anti-VEGF Antibody for the Treatment of Predominantly Classic Choroidal Neovascularization in AMD) studies that clinical trials demonstrated significant improvement in visual outcomes in patients with nAMD in response to anti-VEGF treatment, namely ranibizumab. ${ }^{10,11}$ These trials demonstrated the efficacy of ranibizumab and led to its approval by the FDA in 2006 for the treatment of nAMD. ${ }^{12}$

Despite the success of VEGF suppression as a mainstay of treatment in AMD, there is a clear discrepancy between the successful results reported in randomized controlled trials and patient outcomes seen in clinical practice. ${ }^{13}$ Though the exact cause is unknown, some of this difference can likely be accounted for by differences in real-world prescribing patterns and patient compliance as compared to clinical trials. Current prescribing strategies of anti-VEGF treatment consist of fixed schedule, treat-and-extend (TREX), or pro re nata (PRN). Of these, fixed dosing closely aligns with the experimental conditions of early clinical trials that demonstrated the benefits anti-VEGF injections could achieve. Though effective, frequent monitoring and injection visits represent a heavy financial and psychosocial burden on patients, leading to physicians prescribing less rigorous treatment schedules compared to randomized control trials. ${ }^{14-16}$ Furthermore, intravitreal injections carry inherent risk and benefit of improved vision from increased injection frequency of current drugs must be weighed with potential complications. ${ }^{17}$ Given these challenges of current anti-VEGF treatment, researchers have been investigating how to achieve optimum visual outcomes while decreasing treatment frequency and burden on patients.

One promising solution to greatly reduce injection frequency and maintain pharmacological efficacy is an implantable device that can deliver the controlled release of VEGF neutralizing agents. ${ }^{18}$ The most promising implant that is undergoing late phase clinical trials is the ranibizumab port delivery system (PDS), a small, refillable eye implant that dispenses ranibizumab. This review covers the current evidence to date of the therapeutic potential of the ranibizumab port delivery system in the treatment of nAMD.

\section{Ranibizumab Port Delivery System (PDS)}

The port delivery system (PDS) is a small, durable, refillable reservoir designed to sustainably release ranibizumab directly into the vitreous cavity for treatment of nAMD. It is surgically implanted via a specialized tool through an incision in the sclera and pars plana. The implant is designed with four main components: an extrascleral flange to seat the PDS in the sclera, a self-sealing septum in the flange for drug replenishment, the reservoir body for storing the concentrated drug, and a porous metal release element allowing for the drug to passively diffuse via a concentration gradient directly into the vitreous cavity. ${ }^{19}$ The PDS is currently designed to utilize a customised formulation of ranibizumab, which differs from the currently used formulation approved by the FDA for intravitreal injection. ${ }^{20}$

The medication to be delivered by the PDS platform, ranibizumab, is a $48.3 \mathrm{kD}$ monoclonal antibody fragment designed to bind and neutralize all isoforms of VEGF-A. ${ }^{21}$ It is currently approved as a $0.5 \mathrm{mg}$ intravitreal injection and was approved by the FDA in 2016 as a prefilled syringe of the same dosage for the treatment of nAMD. ${ }^{22}$ In the Phase II clinical trials, formulations of $10 \mathrm{mg} / \mathrm{mL}$, $40 \mathrm{mg} / \mathrm{mL}$, and $100 \mathrm{mg} / \mathrm{mL}$ were used in the PDS with the $100 \mathrm{mg} / \mathrm{mL}$ formulation being utilized exclusively in the Phase III trials. ${ }^{23-25}$

\section{Clinical Trials Phase I}

A Phase I trial was conducted by ForSight Vision4 to test the safety of the ranibizumab PDS. ${ }^{26,27}$ Twenty treatmentnaive patients with neovascular AMD were implanted with a PDS prefilled with $250 \mu \mathrm{g}$ of ranibizumab and were followed monthly for treatment for a year. The device would be refilled based on predetermined visual acuity and optical coherence tomography criteria with $250 \mu \mathrm{g}$ of ranibizumab injected into the implant and an $250 \mu \mathrm{g}$ intravitreal ranibizumab bolus.

The primary outcome was incidence and severity of adverse events (AEs). The primary endpoint was at 1 year, followed by an observation period of 24 months. Secondary outcomes included visual acuity and anatomical findings.

For the primary outcomes, there were 77 AEs reported, conjunctival hyperemia being most common. Four of these patients had AEs considered serious and were all related to device placement. These complications were endophthalmitis, traumatic cataract, and two cases of persistent vitreous hemorrhage. Of these cases, 3 of the 4 patients showed improvement in visual acuity over baseline at 12 months. The mean number of refills was 4.8 in 12 months.

For secondary outcomes, the average improvement in visual acuity for the twenty patients was approximately 
10 ETDRS letters at 12 months, with 10 of the 20 patients improving by at least three lines and two patients losing at least three lines. A mean decrease in central retinal thickness was observed for the cohort as well. Planned explantation of the PDS in six patients at 12 months demonstrated functional and intact devices consistent with the unimplanted PDS, with the implanted PDS being well tolerated by the remaining 14 patients transitioned to intravitreal injections during the observation period.

The study met its primary endpoint of demonstrating the feasibility of the ranibizumab PDS.

\section{Phase II - Long-Acting Delivery of Ranibizumab (LADDER) Trial}

A 38-month Phase II randomized, multicenter, active treatment-controlled, dose-ranging study was conducted by Genentech to assess the safety and effectiveness of the ranibizumab PDS for the treatment of nAMD. ${ }^{19,23}$ A total of 220 treatment responsive patients with newly diagnosed nAMD - defined by a diagnosis within 9 months of screening and a decrease of central foveal thickness (CFT) of $50 \mu \mathrm{m}$ or stable/improved bestcorrected visual acuity (BCVA) after anti-VEGF treatment initiation - were included in the study. The study was designed with four treatment arms in which patients were randomized to a 3:3:3:2 ratio of PDS filled with $10 \mathrm{mg} /$ $\mathrm{mL}, 40 \mathrm{mg} / \mathrm{mL}$, or $100 \mathrm{mg} / \mathrm{mL}$ formulations of ranibizumab or monthly $0.5 \mathrm{mg}$ intravitreal ranibizumab injections. Providers and patients were masked with exception for assignment to either the PDS arms or the monthly intravitreal injection arms.

Patients were assessed monthly for standard safety and functional outcomes (BCVA, CFT, etc.). Additional assessments for PDS patients occurred after device insertion and implant refills. The PDS was refilled pro re nata based on criteria corresponding to nAMD disease progression. These criteria include an increase in CFT of 75 or more $\mu \mathrm{m}$ compared to the average CFT of the last two visits, increase of 100 or more $\mu \mathrm{m}$ of the lowest CFT for that patient, decrease in BCVA of 5 or more ETDRS letters compared to the average CFT of the last two visits, decrease in BCVA of 10 or more ETDRS letters compared to the patient's highest BCVA, or presence of new retinal hemorrhage. PDS patients were treated with open-label $0.5 \mathrm{mg}$ intravitreal ranibizumab as rescue therapy if lack of clinical efficacy criteria were met or worsening disease did not respond to PDS refills. $22.4 \%, 4.8 \%$, and $1.7 \%$ of the $10 \mathrm{mg} / \mathrm{mL}, 40 \mathrm{mg} / \mathrm{mL}, 100 \mathrm{mg} / \mathrm{mL}$ PDS patients, respectively, required rescue therapy.

The primary efficacy outcome of the study was time to first implant refill. Patients were enrolled in the study on average of $16.8 \pm 6$ months (mean $\pm \mathrm{SD}$ ) for subjects in the PDS arms and $16.4 \pm 6.8$ months for the monthly $0.5 \mathrm{mg}$ intravitreal ranibizumab injections patients. For the primary outcomes, the highest concentration PDS group demonstrated the longest median time to implant refill. The median time for implant refill in the PDS $10 \mathrm{mg} / \mathrm{mL}, 40 \mathrm{mg} / \mathrm{mL}$, and $100 \mathrm{mg} / \mathrm{mL}$ arms was 8.7, 13.0, and 15.0 months, respectively. There was statistically significant difference between the median time to implant refill between the $10 \mathrm{mg} / \mathrm{mL}$ and $100 \mathrm{mg} / \mathrm{mL}$ PDS cohorts as well as between the $10 \mathrm{mg} / \mathrm{mL}$ and $40 \mathrm{mg} / \mathrm{mL}$ PDS cohorts, but not between the $40 \mathrm{mg} / \mathrm{mL}$ and $100 \mathrm{mg} / \mathrm{mL}$ PDS arms. $63.5 \%, 71.3 \%$, and $79.8 \%$ of the patients in PDS $10 \mathrm{mg} / \mathrm{mL}, 40 \mathrm{mg} / \mathrm{mL}$, and $100 \mathrm{mg} / \mathrm{mL}$ arms did not require an implant refill at 6 months or greater. The mean number of ranibizumab treatments was $3.7 \pm 6.7$, $2.6 \pm 2.3,2.4 \pm 1.9$, and $16.8 \pm 6.7$ for the $10 \mathrm{mg} / \mathrm{mL}, 40 \mathrm{mg} /$ $\mathrm{mL}, 100 \mathrm{mg} / \mathrm{mL}$ PDS patients, and monthly $0.5 \mathrm{mg}$ intravitreal ranibizumab injections patients, respectively.

The secondary efficacy outcomes of the trial were functional visual and anatomical outcomes. Patients in the $100 \mathrm{mg} / \mathrm{mL}$ PDS and monthly $0.5 \mathrm{mg}$ intravitreal ranibizumab arms demonstrated visual improvement - the adjusted BCVA from baseline at 9 months was $-3.2,-0.5,+5.0$, and +3.9 ETDRS letters for the $10 \mathrm{mg} / \mathrm{mL}, 40 \mathrm{mg} / \mathrm{mL}, 100 \mathrm{mg} / \mathrm{mL}$ PDS patients, and monthly $0.5 \mathrm{mg}$ intravitreal ranibizumab injections patients, respectively. A post-hoc noninferiority test between the $100 \mathrm{mg} / \mathrm{mL}$ PDS and monthly $0.5 \mathrm{mg}$ intravitreal ranibizumab injections arms demonstrated that the BCVA between the two cohorts were comparable. For anatomical outcomes, the adjusted change in CFT from baseline at 9 months was $+54.4,-0.5,-1.7$, and $-6.3 \mu \mathrm{m}$ for the $10 \mathrm{mg} / \mathrm{mL}, 40 \mathrm{mg} / \mathrm{mL}, 100 \mathrm{mg} / \mathrm{mL}$ PDS patients, and monthly $0.5 \mathrm{mg}$ intravitreal ranibizumab injections patients, respectively.

Ocular adverse events (AEs) were observed more frequently in the PDS arms compared to the monthly $0.5 \mathrm{mg}$ intravitreal ranibizumab arm, with 16 of the 179 of PDS patients $(8.9 \%)$ reporting serious ocular AEs and no serious ocular AEs reported in the monthly intravitreal ranibizumab injection patients. The most common serious AEs were vitreous hemorrhage, occurring in 7 PDS patients (3.9\%). Endophthalmitis occurred in 3 PDS patients, with one case occurring within a month of device implantation. 
These patients were culture negative and the PDS devices were explanted, to which BCVA returned to baseline after disease resolution. Rhegmatogenous retinal detachments occurred in 4 PDS patients, with one event occurring within a month of implant insertion. These were repaired by pneumatic retinopexy or vitrectomy in 2 PDS patients and device explanation and scleral buckle in the other 2 patients. Other serious ocular AEs in the PDS groups in order of descending event incidence include: conjunctival erosion (3), reduced visual acuity (3), conjunctival retraction (2), hypotony (1), retinal hemorrhage (1), retinal tear (1), proliferative retinopathy (1), conjunctival filtering bleb leak (1), hyphema (1), wound secretion (1), and increased IOP (1).

The profiles of systemic adverse events (AEs) between the PDS arms to the monthly $0.5 \mathrm{mg}$ intravitreal ranibizumab arm were comparable, with the percentage of patients with $\geq 1$ systemic AE being $76 \%$ to $79 \%$ in the PDS arms and $78 \%$ in the monthly intravitreal ranibizumab arm. The System Organ Class with the highest incidence was infections and infestations for all treatment arms, with the incidence in the PDS arms ranging from $42 \%$ to $45 \%$ and $49 \%$ in the monthly intravitreal ranibizumab arm. However, there were a few System Organ Class events that were more common in the PDS arms versus the monthly intravitreal ranibizumab arms: gastrointestinal (5-15\% versus $0 \%$ ), nervous system disorders (14-30\% versus 7\%), and injury, poisoning, and procedural complications (17-27\% versus 10\%). The gastrointestinal AEs were considered nonserious with nausea, constipation, and gastroesophageal reflux disease being the most common in the PDS arms. Most nervous system disorders events were also considered nonserious with the most common $\mathrm{AE}$ being headaches in the PDS arms (11-19\%) and the monthly ranibizumab arm (2\%). Of the patients with headache in the PDS arms, 76\% occurred during the perioperative period largely due to discomfort from incision and sutures, but $95 \%$ of these events resolved within a month. Though it is unclear its relation to the PDS directly, there were serious AEs of cerebrovascular accidents in the PDS arms $(0-3.2 \%)$ compared to none in the monthly ranibizumab arm. For the injury, poisoning and procedural complications, these events were not temporally related to PDS implant or procedures and were mostly considered nonserious events including falls, contusions, fractures, and sprains. The only serious AE reported in this category was one instance of a serious fall in the PDS arms compared to none in the monthly ranibizumab arm.
The study met its primary efficacy endpoint, leading to a Phase III trial.

\section{Phase III - ARCHWAY Trial}

A Phase III randomized, multicenter, open-label trial to test the safety and efficacy of the $100 \mathrm{mg} / \mathrm{mL}$ ranibizumab PDS compared to $0.5 \mathrm{mg}$ ranibizumab intravitreal injections for nAMD treatment has completed patient enrollment of 418 patients and is currently ongoing. ${ }^{24}$ PDS patients will receive refill/exchanges at fixed 24-week intervals while patients in the comparator arm will receive monthly 0.5 ranibizumab intravitreal injections. The primary outcome is change in best-corrected visual acuity (BCVA) from baseline by ETDRS VA averaged over weeks 36 to 40 and the trial is expected to be completed by April 2021.

\section{Phase III Extension Study - PORTAL Trial}

A Phase III non-randomized, multicenter, open-label extension study to evaluate the long-term tolerability and safety of the $100 \mathrm{mg} / \mathrm{mL}$ ranibizumab PDS has been initiated by Genentech and is planning to enroll an estimated 500 nAMD patients from the LADDER and ARCHWAY studies. ${ }^{25}$ PDS patients will receive refill/exchanges at fixed 24-week intervals, like the ARCHWAY trial, for a duration of 144 weeks. The primary outcomes will be the incidence and severity of both ocular and systemic adverse events and are expected to be completed by January 2022 .

\section{Discussion}

Evidence to date suggests that the ranibizumab port delivery system (PDS) represents a promising delivery platform to reduce treatment burden and improve visual outcomes in patients with nAMD. Given the potential of monthly ranibizumab intravitreal injections exhibited from the MARINA and ANCHOR trials, a sustained release of a well-studied medication like ranibizumab represents a desirable target. ${ }^{10,11}$ Indeed, the results from the LADDER trial demonstrate that the PDS with the $100 \mathrm{mg} / \mathrm{mL}$ formulation is noninferior to monthly intravitreal injections of ranibizumab in terms of visual and anatomical outcomes, leading to the ongoing Phase III trials. $^{19}$

The encouraging results of the LADDER study must be tempered by the differences in safety of the PDS relative to the current standard of practice. No procedure is riskfree, and frequent intravitreal injections carries its own cumulative risk of adverse events and patient burden. ${ }^{14}$ 
However, the use of PDS requires a surgical procedure, which greatly contributed to the observed serious adverse events were observed in the PDS arms but not in the intravitreal injection arm of the trial. Additionally, there was a higher rate of systemic adverse events in the PDS arms, specifically gastrointestinal and nervous system disorders, compared to the monthly intravitreal ranibizumab arm. These events were mainly considered non-serious, like nausea and headaches, that correspond largely to the postoperative period. As such, the PDS has gone through some optimization to help mitigate some of these risks, such as improving the implant insertion procedure with laser ablation of the pars plana at the scleral incision site, diminishing the rate of vitreous hemorrhage. ${ }^{19,28}$ The ongoing Phase III trials should elucidate the safety profile of the PDS further.

It is important to mention the alternatives in extended dosing treatments in the discussion of the PDS. As mentioned previously, the currently available anti-VEGF treatments include intravitreal injections of pegaptanib, bevacizumab, ranibizumab, aflibercept, and brolucizumab. There have been several trials investigating the potential of extended dosing trials with several of these medications to reduce treatment burden. Of note, the randomized Phase 4 ALTAIR trial demonstrated a last intravitreal aflibercept injection interval of at least 12 weeks with a 2 or 4 week treat-and-extend (TREX) strategy in 57\% and $60 \%$ of treatment-naive patients with nAMD by week $96 .{ }^{29}$ The randomized Phase 3 trials of HAWK and HARRIER demonstrated that approximately $50 \%$ of patients with untreated, active nAMD can be maintained on 12 week interval treatments of intravitreal brolucizumab without rescue therapy. ${ }^{30}$ From clinical practice and published data, the q12 week injection intervals for aflibercept and brolucizumab are the longest demonstrated efficacious duration of the currently available medications.

Upcoming intravitreal anti-VEGF therapies are also being evaluated for extended VEGF antagonism including abicipar pegol (Allergan, Dublin, Ireland), conbercept (Chengdu Kanghong Biotechnology, Sichuan, China), KSI-301 (Kodiak Sciences, Palo Alto, CA, USA), and faricimab (Genentech, San Francisco, CA, USA). Abicipar is a designed ankyrin repeat protein anti-VEGF drug demonstrated efficacy at 12-week dosing in the CEDAR/SEQUOIA trials, but also had a high incidence of intraocular inflammation with $15 \%$ in the abicipar group compared to $<1 \%$ in the ranibizumab arm in both trials. ${ }^{31}$ Conbercept is an anti-VEGF fusion protein that has reported efficacy with quarterly injections after three monthly injections in the PHOENIX trial in China and is currently ongoing the phase 3 PANDA-1 trial in the United States. ${ }^{32,33}$ KSI-301 is an antibody biopolymer conjugate that demonstrated efficacy at 16 weeks in a small Phase $1 \mathrm{~b}$ study of 35 patients with nAMD, with the Phase 2 DAZZLE study to test its efficacy at 12, 16, and 20 months. ${ }^{34,35}$ Faricimab is a bispecific antibody for VEGF and angiopoietin 2 that showed efficacy at 16 weeks in the phase II STAIRWAY trial. ${ }^{36}$ These emerging therapies all represent ongoing investigations for improving the durability of anti-VEGF antagonism and should be compared as potential alternatives to the ranibizumab PDS as ongoing investigations and results emerge.

The benefit of VEGF antagonism in the treatment of nAMD is clear, but VEGF is also a critical neurotrophic factor that has been shown to play a critical role in the development and maintenance of retinal vasculature. ${ }^{37}$ Potent, extended neutralization of VEGF may be disruptive to the health of neurovascular cells and as an example, there is some evidence that there is an increased risk of geographic atrophy in patients treated monthly as opposed to patient treated pro re nata. ${ }^{38,39}$ Any of the extended durability anti-VEGF treatments on the horizon, including the ranibizumab PDS, may increase the potential adverse events associated with the prolonged antagonism of VEGF and should be considered as one of the clinical disadvantages of pursuing this avenue of nAMD treatment.

The ranibizumab port delivery system appears to offer the efficacy seen in clinical trials combined with a decreased need for frequent intravitreal injections. This represents a welcomed advancement in the drive to reduce patient burden in the treatment of nAMD, and may be an attractive option for patients given positive results of the upcoming Phase III trials.

\section{Disclosure}

Peter K. Kaiser is a consultant for Alcon, Allergan, Allegro, Bayer, Regeneron, and Novartis. He also reports personal fees from Bayer, Regeneron, Novartis, Genentech, Kanghong, Allergan, and Kodiak, during the conduct of the study. The authors report no other conflicts of interest in this work.

\section{References}

1. Chopdar A, Chakravarthy U, Verma D. Age related macular degeneration. BMJ. 2003;326(7387):485-488. doi:10.1136/bmj.326. 7387.485 
2. Lim LS, Mitchell P, Seddon JM, Holz FG, Wong TY. Age-related macular degeneration. The Lancet. 2012;379(9827):1728-1738. doi:10.1016/S0140-6736(12)60282-7

3. Schmidt-Erfurth U, Chong V, Loewenstein A, et al. Guidelines for the management of neovascular age-related macular degeneration by the European Society of Retina Specialists (EURETINA). $\mathrm{Br}$ $J$ Ophthalmol. 2014;98(9):1144-1167. doi:10.1136/bjophthalmol2014-305702

4. Klein R, Chou C-F, Klein BEK, Zhang X, Meuer SM, Saaddine JB. Prevalence of age-related macular degeneration in the US population. Arch Ophthalmol. 2011;129(1):75-80. doi:10.1001/archophthalmol. 2010.318

5. Miller JW. Age-related macular degeneration revisited - piecing the puzzle: the LXIX Edward Jackson Memorial Lecture. Am J Ophthalmol. 2013;155(1):1-35.e13. doi:10.1016/j.ajo.2012.10.018

6. Agarwal A, Rhoades WR, Hanout M, et al. Management of neovascular age-related macular degeneration: current state-of-the-art care for optimizing visual outcomes and therapies in development. Clin Ophthalmol Auckl NZ. 2015;9:1001-1015. doi:10.2147/OPTH. S74959

7. Gragoudas ES, Adamis AP, Cunningham ET, Feinsod M, Guyer DR, VEGF Inhibition Study in Ocular Neovascularization Clinical Trial Group. Pegaptanib for neovascular age-related macular degeneration. N Engl J Med. 2004;351(27):2805-2816. doi:10.1056/NEJMoa042760

8. Rosenfeld PJ, Moshfeghi AA, Puliafito CA. Optical coherence tomography findings after an intravitreal injection of bevacizumab $\left(\right.$ Avastin ${ }^{\circledR}$ ) for neovascular age-related macular degeneration. Ophthalmic Surg Lasers Imaging Retina. 2005;36(4):331-335. doi:10.3928/1542-8877-20050701-14

9. Michels S, Rosenfeld PJ, Puliafito CA, Marcus EN, Venkatraman AS. Systemic bevacizumab (Avastin) therapy for neovascular age-related macular degeneration: twelve-week results of an uncontrolled open-label clinical study. Ophthalmology. 2005;112(6):1035-1047. e9. doi:10.1016/j.ophtha.2005.02.007

10. Rosenfeld PJ, Brown DM, Heier JS, et al. Ranibizumab for neovascular age-related macular degeneration. $N$ Engl J Med. 2006;355 (14):1419-1431. doi:10.1056/NEJMoa054481

11. Brown DM, Kaiser PK, Michels M, et al. Ranibizumab versus verteporfin for neovascular age-related macular degeneration. $N$ Engl J Med. 2006;355(14):1432-1444. doi:10.1056/NEJMoa062655

12. Drug Approval Package: Lucentis (Ranibizumab). Available from: https://www.accessdata.fda.gov/drugsatfda_docs/nda/2006/ 125156s0000_LucentisTOC.cfm. Accessed February. 14, 2020.

13. Chong V. Ranibizumab for the treatment of wet AMD: a summary of real-world studies. Eye Lond Engl. 2016;30(2):270-286. doi:10.1038/ eye.2015.217

14. Boyle J, Vukicevic M, Koklanis K, Itsiopoulos C, Rees G. Experiences of patients undergoing repeated intravitreal anti-vascular endothelial growth factor injections for neovascular age-related macular degeneration. Psychol Health Med. 2018;23 (2):127-140. doi:10.1080/13548506.2016.1274040

15. Holekamp NM, Liu Y, Yeh W-S, et al. Clinical utilization of anti-VEGF agents and disease monitoring in neovascular age-related macular degeneration. Am J Ophthalmol. 2014;157 (4):825-833.e1. doi:10.1016/j.ajo.2013.12.018

16. Rao P, Lum F, Wood K, et al. Real-world vision in age-related macular degeneration patients treated with single anti-VEGF drug Type for 1 year in the IRIS registry. Ophthalmology. 2018;125 (4):522-528. doi:10.1016/j.ophtha.2017.10.010

17. Ghasemi Falavarjani K, Nguyen QD. Adverse events and complications associated with intravitreal injection of anti-VEGF agents: a review of literature. Eye. 2013;27(7):787-794. doi:10.1038/eye.2013.107

18. Schlottmann PG, Alezzandrini AA, Zas M, Rodriguez FJ, Luna JD, $\mathrm{Wu}$ L. New treatment modalities for neovascular age-related macular degeneration. Asia-Pac J Ophthalmol. 2017;6(6):514-519. doi:10. 22608/APO.2017258
19. Campochiaro PA, Marcus DM, Awh CC, et al. The port delivery system with ranibizumab for neovascular age-related macular degeneration: results from the randomized Phase 2 ladder clinical trial. Ophthalmology. 2019;126(8):1141-1154. doi:10.1016/j.ophtha.2019. 03.036

20. Genentech: Press Releases | Wednesday, July 25, 2018. Available from: https://www.gene.com/media/press-releases/14739/2018-0725/genentech-unveils-positive-phase-ii-resu. Accessed February 18, 2020.

21. Ferrara N, Damico L, Shams N, Lowman H, Kim R. Development of ranibizumab, an anti-vascular endothelial growth factor antigen binding fragment, as therapy for neovascular age-related macular degeneration. Retina Phila Pa. 2006;26(8):859-870. doi:10.1097/ 01.iae.0000242842.14624.e7

22. Lucentis (ranibizumab) FDA Approval History. Drugs.com. Available from: https://www.drugs.com/history/lucentis.html. Accessed February 15,2020 ..

23. Study of the Efficacy and Safety of the Ranibizumab Port Delivery System (RPDS) for Sustained Delivery of Ranibizumab in Participants With Subfoveal Neovascular Age-Related Macular Degeneration (AMD) (LADDER) ClinicalTrials.gov. Available from: https://clinical trials.gov/ct2/show/NCT02510794. Accessed February 18, 2020.

24. A Phase III study to evaluate the port delivery system with ranibizumab compared with monthly ranibizumab injections in participants with wet age-related macular degeneration (Archway). ClinicalTrials. gov. Available from: https://clinicaltrials.gov/ct2/show/ NCT03677934. Accessed February 25, 2020..

25. Extension Study for the Port Delivery System With Ranibizumab (Portal) ClinicalTrials.gov. Available from: https://clinicaltrials.gov/ ct2/show/NCT03683251. Accessed February 27, 2020.

26. Laganovska G First-in-human results of a refillable drug delivery implant providing release of ranibizumab in patients with neovascular AMD. Presented at the: American Academy of Ophthalmology, Retina Subspecialty Day; November 9, 2012; Chicago, IL.

27. Rubio RG. Long-acting anti-VEGF delivery. Retina Today. 2014;78-80.

28. Bantseev V, Schuetz C, Booler HS, et al. Evaluation of surgical factors affecting vitreous hemorrhage following port delivery system with ranibizumab implant insertion in a minipig model. Retina Phila Pa. 2019:1. doi:10.1097/IAE.0000000000002614.

29. Ohji M, Takahashi K, Okada AA, et al. Efficacy and safety of intravitreal aflibercept treat-and-extend regimens in exudative age-related macular degeneration: 52- and 96-week findings from ALTAIR. $A d v$ Ther. 2020;37(3):1173-1187. doi:10.1007/s12325-020-01236-x

30. Dugel PU, Koh A, Ogura Y, et al. HAWK and HARRIER: Phase 3, multicenter, randomized, double-masked trials of brolucizumab for neovascular age-related macular degeneration. Ophthalmology. 2020;127(1):72-84. doi:10.1016/j.ophtha.2019.04.017

31. Khurana R Safety and efficacy of abicipar in patients with neovascular age-related macular degeneration. Presented at the: American Academy of Ophthalmology, Retina Subspecialty Day; October 26, 2018; Chicago, IL.

32. Liu K, Song Y, Xu G, et al. Conbercept for treatment of neovascular age-related macular degeneration: results of the randomized Phase 3 PHOENIX study. Am J Ophthalmol. 2019;197:156-167. doi:10.1016/ j.ajo.2018.08.026

33. Efficacy and Safety Trial of Conbercept Intravitreal Injection for Neovascular Age-related Macular Degeneration (PANDA-1) - Full Text View - ClinicalTrials.gov. Available from: https://clinicaltrials. gov/ct2/show/NCT03577899. Accessed April 7, 2020.

34. Wykoff $\mathrm{C}$ Extended durability in exudative retinal diseases using the novel intravitreal anti-VEGF antibody biopolymer conjugate KSI-301: results from the Phase $1 \mathrm{~b}$ study in patients with AMD, DME and RVO. Presented at the: American Academy of Ophthalmology, Retina Subspecialty Day; October 11, 2019; San Francisco, CA. 
35. A study to evaluate the efficacy and safety of KSI-301, an anti-VEGF antibody biopolymer conjugate, versus aflibercept in patients with neovascular (Wet) age-related macular degeneration. - Full text view - ClinicalTrials.gov. Available from: https://clinicaltrials.gov/ct2/ show/NCT04049266. Accessed April 7, 2020..

36. Csaky K Data supporting the sustained efficacy of faricimab, a bispecific antibody neutralizing both angiopoietin-2 and VEGF-A. Presented at the: American Academy of Ophthalmology, Retina Subspecialty Day; October 12, 2019; San Francisco, CA.
37. Usui Y, Westenskow PD, Kurihara T, et al. Neurovascular crosstalk between interneurons and capillaries is required for vision. J Clin Invest. 2015;125(6):2335-2346. doi:10.1172/JCI80297

38. Sadda SR, Tuomi LL, Ding B, et al. Macular atrophy in the HARBOR study for neovascular age-related macular degeneration. Ophthalmology. 2018;125(6):878-886. doi:10.1016/j.ophtha.2017.12.026

39. Chakravarthy U, Harding SP, Rogers CA, et al. Alternative treatments to inhibit VEGF in age-related choroidal neovascularisation: 2-year findings of the IVAN randomised controlled trial. The Lancet. 2013;382(9900):1258-1267. doi:10.1016/S0140-6736(13)61501-9

\section{Publish your work in this journal}

Clinical Ophthalmology is an international, peer-reviewed journal covering all subspecialties within ophthalmology. Key topics include: Optometry; Visual science; Pharmacology and drug therapy in eye diseases; Basic Sciences; Primary and Secondary eye care; Patient Safety and Quality of Care Improvements. This journal is indexed on PubMed

Submit your manuscript here: https://www.dovepress.com/clinical-ophthalmology-journal
Central and CAS, and is the official journal of The Society of Clinical Ophthalmology (SCO). The manuscript management system is completely online and includes a very quick and fair peer-review system, which is all easy to use. Visit http://www.dovepress.com/ testimonials.php to read real quotes from published authors. 